\title{
Parry-Romberg Syndrome with Band-like Alopecia
}

Sir,

Progressive hemifacial atrophy (HFA), also known as Parry-Romberg syndrome is characterized by progressive wasting of the face, including the subcutaneous tissue, fat, cartilage and bone. ${ }^{1}$ The aim of the present communication is to report on an interesting case of Parry Romberg syndrome with a peculiar finding of "band-like alopecia". A 12-year-old boy presented with progressive painless loss of scalp hair in a peculiar band like fashion for the last 5 years. (Fig.1). On examination the hair loss, which was non-scarring in nature took place in a longitudinal, band-like fashion measuring about $2 \mathrm{~cm}$ in width with variable length. The margins of the alopecia had easily pluckable hairs. The hairs however, had normal colour and lustre.

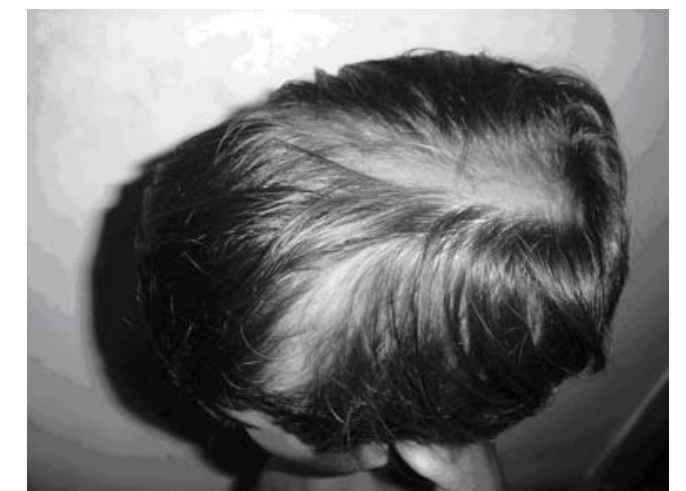

Fig. 1 Longitudinal, band-like alopeicia seen over the parietal and temporal regions.

He also had progressive wasting of the left half of the face with exaggerated ipsilateral nasolabial furrow and prominent eminence for the last 1 year (Fig.2). There was no history of drooling or inability to close the eyes, weakness in the limbs, seizures or headache. No history could be elicited relating to facial trauma, rash or joint pain. The skin of the left half of the face appeared taut and shiny without pigmentary changes.

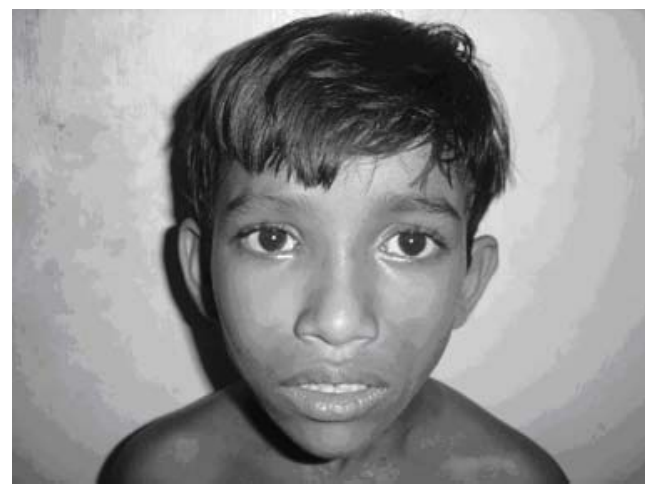

Fig. 2 Mild wasting of the hemiface with exaggerated nasolabial furrow and prominent zygomatic eminence.
No other area of localized subcutaneous tissue atrophy, hyperpigmentation or vitiligo was noted. The neck, trunk and limbs were symmetrical. The rest of the neurological examination was normal.

Hematological examinations including liver and kidney function tests, blood sugar and lipid profile were normal. Anti-nuclear antibody titre was positive at 1:80 dilution. Ultrasound examination of the abdomen was normal. CT scan of the head and paranasal sinuses were normal.

HFA may rarely present at birth but more commonly do so in early childhood. ${ }^{1}$ The eyelashes, eyebrows and scalp hair on the involved side may be affected and ipsilateral alopecia may occur. ${ }^{2}$ Although alopecia is commonly seen, the distinct morphology of the longitudinal band like form as evident in the present case has not been described with this condition.

Neurological manifestation seen includes recurrent headaches, contralateral partial seizures, masticatory spasms, cognitive abnormalities and hemiparesis. ${ }^{3}$ Cutaneous abnormalities described include pigmentary changes, vitiligo, nevi and hyperpigmented areas on the affected side. Ophthalmological manifestations may include ipsilateral Horner's syndrome, blepharophimosis, chronic cyclitis, iritis, cataract and secondary glaucoma. In lipodystropy, the involvement is bilateral and spares cartilage and bone; in scleroderma, the skin becomes cold, waxy and inelastic and adheres to the subcutaneous tissue. ${ }^{1}$

\section{Rajoo Thapa, Apurba Ghosh and Sandipan Dhar}

Rajoo Thapa, MD.

The Department of Pediatrics, The Institute of Child Health,

11, Dr. Biresh Guha Street, Kolkata-700017, West Bengal, India. Phone: +919868780960 Fax: +913322905686. E-mail: rajoothapa@yahoo.co.in.

\section{REFERENCES}

1. Menkes JH. Progressive Facial Hemiatrophy (ParryRomberg syndrome). In Menkes JH, Sarnat HB, eds. Child Neurology, $6^{\text {th }}$ ed. Philadelphia; Lippincott William \& Wilkins, 2000;210-211.

2. Wartenberg R. Progressive facial hemiatrophy. Arch Neurol Psychiat 1945;54: 75-96.

3. Berg BO. Parry-Romberg syndrome. In Swaiman KF, Ashwal S, eds. Pediatric Neurology-Principles and Practice, $3^{\text {rd }}$ ed. Missouri; Mosby, 1999;543. 